\title{
Approaches to Health Research Priority Setting in the Philippines across the Years
}

\author{
Alain Jason A. Generale, ${ }^{1}$ Reneepearl Kim P. Sales, ${ }^{1}$ Teddy S. Dizon ${ }^{1}$ and Alan B. Feranil ${ }^{2}$ \\ ${ }^{1}$ Alliance for Improving Health Outcomes \\ ${ }^{2}$ Research Agenda Committee, Philippine National Health Research System
}

\begin{abstract}
Background. As one of the research councils under the Department of Science and Technology (DOST), health research priority setting has been the mandate of the Philippine Council for Health Research and Development (PCHRD) since its establishment in 1982. The development of the National Unified Health Research Agenda (NUHRA) convenes the major stakeholders for health in the country to establish the priorities for health research. The NUHRA aims to address the most urgent health issues in the country for the generation of solutions to the health concerns of the country.
\end{abstract}

Objectives and Method. Through document review, this paper describes the approaches and lessons learned in research priority setting since the establishment of the Philippine National Health Research System.

Results. The Philippines has employed a bottoms-up, top-down, and a combination of both approaches to develop its health research agenda.

Conclusion. The health research agenda-setting must consider evolving funding sources, its link to production of researches with high probability of knowledge translation to health technology innovation, and policy formulation. Measuring the impact of the NUHRA to the health systems and health situation of the country is a difficult assessment, but the gradual change in healthcare technology utilization and evidence-informed policies towards health equity can be a subjective measurement of the NUHRA's success.

Key Words: research priorities, support of research, research and development, research agenda

\section{INTRODUCTION}

As one of the research councils under the Department of Science and Technology (DOST), health research priority setting has been the mandate of the Philippine Council for Health Research and Development (PCHRD) since its establishment in 1982. The development of the National Unified Health Research Agenda (NUHRA) convenes the major stakeholders for health in the country to establish the priorities for health research. The NUHRA aims to address the most urgent health issues in the country for the generation of solutions to the health concerns of the country.

The health system operates on a multi-tiered system influenced by broad socio-political factors in national and international contexts. The system maintains a balance between the perspectives of service delivery, resource

Corresponding author: Reneepearl Kim P. Sales, RMT, MLS(ASCPi)CM, MSc

Alliance for Improving Health Outcomes

62 West Avenue, Quezon City 1104, Philippines

Email: kimsales@aiho.org.ph allocation, policies, and research. There is a growing recognition of the influence of health and economic trends in the global context, including multilateral and bilateral organizations that affect health research funding. With the continued influence of international donor agencies 
commissioning for health research, low and middleincome countries (LMIC) are challenged by the specific research mandates of donor agencies which may not be aligned with national health priorities. ${ }^{1}$ This research may be further challenged, as in the Philippines, by its current environment of local governance, geographical, and political jurisdiction that may contribute to fragmentation of health research priorities.

National health research priorities from the 1980s to the mid-1990s were mainly drawn by experts based in the National Capital Region. ${ }^{1}$ In 1999, in response to a call for a multi-stakeholder and participatory formulation of the health research priorities, ${ }^{2}$ a bottoms-up approach to health priority setting was initiated by PCHRD. This approach involved region-based consultations and the elevation of regional priorities to the national level. ${ }^{1}$ This priority setting approach was a precursor to the first NUHRA of the Philippine National Health Research System.

Through document review, this paper describes the approaches and lessons learned in research priority setting since the establishment of the Philippine National Health Research System.

\section{NUHRA 2006-2010}

The formulation of the NUHRA 2006-2010 was anchored on national and local initiatives which included the Millennium Development Goals, Medium-Term Philippine Development Plan 2004-2010, the Fourmula One for Health as Framework for Health Reforms by the Department of Health, and National Science and Technology Plan 20022010. ${ }^{3}$ Inspired by its 1998 precursor, the priority-setting for NUHRA 2006-2010 utilized a bottoms-up approach that engaged multisectoral stakeholders through regional and national consultations. Regions were clustered into four zones: North Luzon, South Luzon, Visayas, and Mindanao. A task force was then convened to formulate a set of national priorities generated from the regional health research priorities. The stakeholders for both the regional and national consultations included the agriculture, environment, and labor sectors as well as non-government organizations. ${ }^{1}$ The process resulted in 422 priorities with six broad health research categories: health financing, governance, health regulations, health service delivery, health technology development, and health research ethics. ${ }^{4}$

There were sustained efforts to advocate for the implementation of NUHRA 2006-2010 because of the participation of major stakeholders. This included advocacy planning in the regional units prior to the dissemination of the research agenda. At the national level, the research agenda was disseminated to various national line agencies and conferences with other donor agencies in the National Capital Region. ${ }^{5}$

The involvement of multisectoral stakeholders resulted in a highly participative and multi-disciplinary consultative process. ${ }^{1}$ As health encompasses disciplines beyond human biology, the inclusion of different sectors addressed health as a multi-faceted phenomenon. Stakeholders appreciated their participation in the consultation and realized the importance of their sector to health in a broader lens. In addition, delegation of region-based conveners and experts ensured that health research priorities were sensitive to local health needs, cultural practices, and available resources.

The bottoms-up approach had its limitations. The region-based conveners and experts had to validate their data due to the limited availability of reliable and accurate epidemiological data at that time. This resulted in the difficulty of some regions to set their health scenario and identify priority health concerns. ${ }^{1}$ While the process was multisectoral, there was limited participation of medical societies, health professionals, the industry, and private sector. $^{6}$ There was difficulty in translating health problems identified into health research priorities. Stakeholders were more familiar with identifying health problems, which were beyond the limited bounds of research. ${ }^{1}$ The integration of all regional inputs and translating them into national health priorities was also a difficult task due to the numerous health research priorities identified at the regional level. ${ }^{1}$ While the list of research priorities were relevant to the country's health situation, the 422 topics identified were too many for the limited resources available for research. ${ }^{6}$ As a result, the NUHRA 2006-2010 was updated into the NUHRA 2008-2010 to refocus the research priorities to better address existing health problems. ${ }^{7}$

Assessment of the NUHRA 2006-2010 revealed that advocacy and dissemination of NUHRA 2006-2010 was assessed as inadequate because several groups in the health research community and other sectors were not aware of or knowledgeable about it. Only one fifth or 96 of the 422 health research priorities were reportedly studied. Sixty two of these 96 researches were completed. ${ }^{6}$

\section{NUHRA 2011-2016}

The framework of the NUHRA 2011-2016 was anchored towards the Aquino Health Agenda, Philippine Development Plan 2011-2016, National Research \& Development Priorities Plan 2011-2016, DOST's FivePoint Priority Program 2011-2016, and the Millennium Development Goals. Unlike its predecessor, the NUHRA 2011-2016 used a top-down approach with the intent of focusing on research priorities with greater assurance of funding and commitment. ${ }^{8}$ The result was a consolidation of the research priorities of the four core agencies of the PNHRS: Department of Health (DOH), DOST, Commission on Higher Education (CHED), and University of the Philippines-National Institutes of Health (UP-NIH). This resulted in 56 topics with four broad categories: health technology development, health financing, health service delivery, and socio-environmental health concerns. 
There were no public dissemination or advocacy activities for NUHRA 2011-2016. However, it was launched during the PCHRD anniversary in 2012 and printed copies were distributed during the PNHRS and DOH National Health Policy Conference. Printed documents were also distributed to the 17 Regional Health Research and Development Consortium (RHRDC). Regions were advised to use the NUHRA 2011-2016 as a guide to develop their regional health research agendas. Overall, it was found that only the core agencies and RHRDCs were made aware of the NUHRA 2011-2016. ${ }^{6}$

A 2015 assessment of the NUHRA 2011-2016 revealed that there was $80 \%$ uptake of the NUHRA 2011-2016, with 45 of the 56 topics studied. A total of 483 NUHRA 2011-2016 researches were funded, amounting to over Php 1.4 million. Of the four broad research categories, health technology development was studied the most (327 studies) while health financing was the least studied category $(25$ studies). PCHRD generated the most NUHRA 2011-2016 research (71\%), while CHED generated the least (4\%). ${ }^{6}$

The research priorities focused on each agency's priorities, dissociating from their interrelationships to improve the health system and attain universal healthcare. Respondents to a focus group discussion on the methodology of the NUHRA 2011-2016 suggested that consultations should be inclusive and consider inputs from the wider health science community. Respondents also recommended the development of an advocacy plan to reach out to the broader research community. Majority of the RHRDCs aligned their agendas with the NUHRA 2011-2016. However, five RHRDCs expressed that the NUHRA 2011-2016 did not reflect the regional situation and priorities and limited the formulation of their regional research agendas. Majority of the RHRDCs recommended region-wide consultations similar to the development of the NUHRA 2006-2010. ${ }^{6}$

\section{NUHRA 2017-2022}

The NUHRA 2017-2022 was guided by the PNHRS Guidelines for Health Research Prioritization. The guideline follows a three-phase framework: 1) integration of information for contextualization of health research issues, mapping of stakeholders, and planning; 2) generation of health research priorities, determining criteria for prioritization, determining methods for deciding on research priorities; and 3) dissemination of the agenda and monitoring and evaluation for research generation and utilization. ${ }^{9}$ Building on the NUHRA 2006-2010 and NUHRA 2011-2016 experience, the developers of the NUHRA 2017-2022 adopted the Kingdon Model for Agenda Setting. The model has three streams: 1) identifying the issues, needs, gaps (problem stream); 2) technical feasibility, knowledge, solutions (policy stream); and 3) national mood, jurisdictional authority, public opinion, and electoral politics (political stream). ${ }^{10}$
Seventeen regional consultations were held which involved stakeholders from the academe, government, hospital, NGO, and private sectors. Each region also developed their respective situational reports describing their stakeholders, health research context, issues, gaps, and threats. In addition, four technical papers were presented to describe the health directions of the country, its current trends, developments, challenges, and the significance of private sector participation in health research. These documents set the background for the regional priority setting exercises. ${ }^{11}$

Prior to the national consultation, thematic analysis was conducted on the inputs from the regional consultations and the DOH and PCHRD national health research priorities following Braun and Clarke's process and iterative categorization. This generated an initial list of six research themes for the NUHRA 2017-2022. The national consultation stakeholders were identified with aid from PNHRS and PCHRD. The stakeholders were presented with the initial list of research priorities and raised their institutional research priorities for inclusion to the NUHRA 2017-2022. The regional research priorities, the PNHRS core agencies' priorities, and the inputs from the national stakeholders were incorporated and synthesized to generate the NUHRA 2017-2022. This resulted in the current research agenda, with 43 research areas clustered into six themes: 1) responsive health systems, 2) researches to enhance and extend healthy lives, 3) holistic approaches to health and wellness, 4) health resiliency, 5) global competitiveness and innovation, and 6) researches in equity and health. ${ }^{11}$

Following the development of the NUHRA 20172022, a draft advocacy and monitoring and evaluation plan was developed in September 2017. The aim of the plan is to systematically and objectively monitor and evaluate the efficiency, effectiveness, and influence of the NUHRA 20172022. It includes indicators for six objectives: advocacy and dissemination, funding, research outputs, collaborations, implementation, and impact.

\section{CONCLUSION}

The Philippines has employed various priority-setting approaches since the first NUHRA was developed in 2006. The bottoms-up, top-down, and combination of the two approaches were utilized to address specific challenges at the time of their conception. All three approaches considered national developments, context, and priorities when formulating the agenda. This is crucial, as a health research agenda aims to allocate limited resources to solve the country's existing and emergent health challenges through research. ${ }^{12}$ The bottoms-up model promoted a multi-disciplinary, inclusive, and participatory approach while integrating regional and national research priorities from the core agencies of the PNHRS. This allowed for the development of a context-sensitive health research agenda 
at the regional level that strengthened the commitment of the stakeholders involved in its development. This approach also led to the non-health sector's greater appreciation for the role they can play in improving the health status of the country.

In NUHRA 2006-2010, the bottoms-up approach yielded over 400 subtopics which led to its revision for a more concise version in 2008. In this approach, it is important to develop a methodology which utilizes regional priorities as inputs to the national consultation with focus. In the development of the NUHRA 2017-2022, overabundance of subtopics was sidestepped by the conduct of thematic analysis on the regional priorities prior to the national consultation. Thus, national level stakeholders were presented with a concise initial list of NUHRA 2017-2022 research themes and subtopics. In addition, the national consultation was only conducted once, as opposed to a series of consultations which could be conducive to over-addition on or over-amendment of the initial list of research priorities.

The top-down model, as utilized in the development of the NUHRA 2011-2016, employed a prescriptive, directive approach to the development of the national and regional health research priorities. This resulted in far fewer subtopics than its predecessor. Assessments revealed that there was $80 \%$ uptake of the NUHRA 2011-2016 topics. While majority of RHRDCs used the NUHRA 2011-2016 as reference for the development of their regional agenda, some RHRDCs found that the NUHRA did not reflect the regional perspective.

In NUHRA 2006-2010 and 2011-2016, inadequate engagement of the private and industry sector was noted. Downplaying the role of these sectors may disintegrate the relationship between the academe, industry, and government. This may result in local technology transfer and utilization to the regions. Without their participation, the private and industry sector may not leverage their strengths in commercialization and utilization of new products, which delays the innovations produced from the health research. ${ }^{13}$

The inclusion of monitoring \& evaluation and information dissemination activities in the planning stage allows for objective assessment of the outcomes of the NUHRA after its conclusion. Nonetheless, the comprehensive and exhaustive list of research areas makes the assessment of the NUHRA a challenge, with a great number of resources competing for funding while there is dearth of fiscal and intellectual resources.

Moving forward, the integrative model for priority setting for health research must consider the evolving funding sources, its link to production of researches with high probability of knowledge translation, and policy formulation. ${ }^{14}$ Measuring the impact of the NUHRA to the health systems and health situation of the country is a difficult assessment, but the gradual change in healthcare technology utilization and evidence-informed policies towards health equity can be a subjective measurement of the NUHRA's success.

\section{Acknowledgments}

The writers thank Prof. Marilyn Ellorin-Crisostomo and Dr. Katherine Villegas-Reyes for their guidance and inputs on this paper.

\section{Statement of Authorship}

All authors approved the final version submitted.

\section{Author Disclosure}

All authors declared no conflict of interest.

\section{Funding Source}

This paper was funded by the Philippine Council for Health Research and Development.

\section{REFERENCES}

1. Feranil A. The bottom up approach in research agenda setting: lessons from the Philippine experience. Global Forum for Health Research, Forum 8; Mexico. 2004.

2. Council for Health Research and Development (COHRED). Essential National Health Research and Priority Setting: Lessons Learned. 1997.

3. Philippine National Health Research System. National Unified Health Research Agenda 2006-2010. 2006.

4. Philippine National Health Research System. National Health Research Agenda 2006-2010. 2006.

5. Feranil A. Going Back to Where It All Started: The Philippine Experience in Priority Setting and Agenda Advocacy. Global Forum for Health Research; Beijing, China. 2007.

6. Ramos-Jimenez P, Aragones D, Mendoza Y, Togonon L, Yubia M, Amita G. Assessment of the 2011-2016 National Unified Health Research Agenda (NUHRA) and the 2011-2015 Regional Unified Health Research Agenda (RUHRA). 2015.

7. Philippine National Health Research System. National Unified Health Research Agenda 2008-2010. Manila, Philippines: PNHRS; 2008.

8. Philippine National Health Research System. National Unified Health Research Agenda 2011-2016. 2011.

9. Philippine National Health Research System. Guidelines for Health Research Prioritization. Manila, Philippines: PNHRS; 2016.

10. Kingdon J. Agendas, Alternatives, and Public Policies. Boston: Little Brown; 1984.

11. Philippine National Health Research System. National Unified Health Research Agenda 2017-2022. 2017.

12. Montorzi G, de Haan S, IJsselmuiden C. Priority Setting for Research for Health: a management process for countries. COHRED. 2010.

13. Farinha L. Triangulation of the triple helix: a conceptual framework. Triple Helix 10th International Conference 2012; West Java, Indonesia. 2012.

14. Tugwell P, Sitthi-Amorn C, Hatcher-Roberts J, Neufeld V, Makara P, Munoz F, et al. Health research profile to assess the capacity of low and middle income countries for equity-oriented research. BMC Public Health. 2006 Jun 12; 6:151. 